\title{
Optimal estimation of unknown data of periodic boundary value problems for first order linear impulsive systems of ordinary. differential equations from indirect noisy observations of their solutions
}

\author{
Nakonechnyi O. G., Podlipenko Yu. K. \\ Taras Shevchenko Kyiv National University, \\ 64/13 Volodymyrska Str., Kyiv, 01601, Ukraine
}

(Received 4 November 2020; Accepted 19 March 2021)

\begin{abstract}
We consider boundary value problems with periodic boundary conditions for first-order linear systems of impulsive ordinary differential equations with unknown right-hand sides and jumps of solutions at the impulse points entering into the statement of these problems which are assumed to be subjected to some quadratic restrictions. From indirect noisy observations of their solutions on a finite system of intervals, we obtain the optimal, in certain sense, estimates of images of their right-hand sides under linear continuous operators. Under the condition that the unknown correlation functions of noises in observations belong to some special sets, it is established that such estimates and estimation errors are expressed explicitly via solutions of special periodic boundary value problems for linear impulsive systems of ordinary differential equations.
\end{abstract}

Keywords: optimal estimates, guaranteed estimates, noisy observations, periodic boundary value problems, impulsive ordinary differential equations.

2010 MSC: $34 \mathrm{H} 05,34 \mathrm{G} 25$

DOI: $10.23939 / \mathrm{mmc} 2021.02 .317$

\section{Introduction}

In the present paper for periodic BVPs for systems of linear impulsive ordinary differential equations, we propose a novel technique of finding optimal estimates of images of their data under linear continuous operators from indirect noisy observations of solutions of these BVPs on a finite system of intervals.

We assume that right-hand sides entering into the statement of these problems and correlation functions of errors in observations are unknown and belong to certain ellipsoids in the corresponding function spaces. Here by observations of unknown solutions we mean functions that are linear transformations of the same solutions distorted by additive random noises.

It is proved that optimal estimates and estimation errors are expressed explicitly via solutions of special uniquely solvable systems of linear impulsive ordinary differential equations with periodic boundary conditions.

To do that, we first solve the problem of guaranteed (minimax) estimation of values of linear functionals from above-mentioned right-hand sides and obtain the boundary value problems, not depending on specific form of linear functionals, that generate the guaranteed estimates as well as the optimal estimates of right-hand sides. Further, we apply these results for obtaining the optimal estimates.

Notice that this work is a continuation of our earlier studies set forth in [1,2], where we elaborate the guaranteed (minimax) estimation method for the case of the problem of estimation of linear functionals from unknown solutions and right-hand sides of first order linear periodic systems of ordinary differential equations.

\section{Preliminaries and auxiliary results}

Let $\mathbb{C}$ denote the field of complex numbers, $\Lambda^{*}$ denote the matrix complex conjugate and transpose of a matrix $\Lambda$. Denote by $X(t)$ a matrix-valued function $X(t)=\left[x_{1}(t), \ldots, x_{n}(t)\right]$ whose columns are 
linearly independent solutions $x_{1}(t), \ldots, x_{n}(t)$ of the homogeneous system

$$
\begin{gathered}
\frac{d x(t)}{d t}=A(t) x(t), \\
\left.\Delta x(t)\right|_{t=t_{i}}=B_{i} x\left(t_{i}\right), \quad i=1, \ldots, q,
\end{gathered}
$$

such that $X(0)=E$, where ${ }^{1} E$ is the unit $n \times n$-matrix, $0=t_{0}<t_{1}<\cdots<t_{q}<t_{q+1}=T$ is a given system of points on the real axis, $A(t)=\left[a_{i j}(t)\right]$ is an $n \times n$-matrix with $a_{i j}(\cdot) \in L^{2}(0, T), B_{i}$ are $n \times n$ constant matrices such that

$$
\operatorname{det}\left(E+B_{i}\right) \neq 0, \quad i=1, \ldots, q .
$$

Further we will assume that the following condition is valid

$$
\operatorname{det}(E-X(T)) \neq 0 \text {. }
$$

Under the conditions (3) and (4) the following nonhomogeneous problem

$$
\begin{aligned}
\frac{d x(t)}{d t} & =A(t) x(t)+B(t) f(t) \quad \text { for a.e. } \quad t \in(0, T), \\
\left.\Delta x(t)\right|_{t=t_{i}} & =B_{i} x\left(t_{i}\right)+C_{i} g_{i}, \quad x(0)=x(T), \quad i=1, \ldots, q,
\end{aligned}
$$

as well as the problem

$$
\begin{gathered}
-\frac{d z(t ; u)}{d t}=A^{*}(t) z(t ; u)+g(t) \quad \text { for a.e. } \quad t \in(0, T), \\
\left.\Delta z(t)\right|_{t=t_{i}}=-\left(E+B_{i}^{*}\right)^{-1} B_{i}^{*} z\left(t_{i}\right)+g_{i}^{\prime}, \quad i=1, \ldots, q, \quad z(0)=z(T),
\end{gathered}
$$

that is adjoint of nonhomogeneous problem (5), (6), are unique solvable for any vector-functions $f(t) \in$ $\mathbb{C}^{r}, g(t) \in \mathbb{C}^{n}$ such that $f \in\left(L^{2}(0, T)\right)^{r}, g \in\left(L^{2}(0, T)\right)^{n}$ and for any vectors $g_{i} \in \mathbb{C}^{k}, g_{i}^{\prime} \in \mathbb{C}^{n}$, where $B(t)=\left[b_{i j}(t)\right]$ is an $n \times r$-matrix with $b_{i j}(\cdot)$ being piecewise continuous on $[0, T], C_{i}$ are $n \times k$ constant matrices.

By a solution, for example of problem (5), (6), we mean a function $x(t) \in \mathcal{A}$ that is left continuous, satisfies the equation (5) almost everywhere (a.e.) on $(0, T)$, and the conditions (6), where by $\mathcal{A}$ we denote a class of functions $y(t) \in \mathbb{C}^{n}$ defined on $[0, T]$ such that $\left.y(\cdot)\right|_{\left(t_{i-1}, t_{i}\right)} \in\left(W_{2}^{1}\left(t_{i-1}, t_{i}\right)\right)^{n}$, $i=1, \ldots, q+1$. Here $W_{2}^{1}(a, b)$ is the space of functions absolutely continuous on an interval $(a, b)$ for which the derivative that exists almost everywhere on $(a, b)$ belongs to space $L^{2}(a, b)$. These assertions follow from the results contained in [3-5].

\section{Statement of the problem of guaranteed estimation of linear functionals defined on unknown data of periodic boundary value problems}

Let $\Omega_{j}^{i}, j=1, \ldots, M_{i}$ be a given system of subintervals of $\left(t_{i-1}, t_{i}\right), F:=\left(f, g_{1}, \ldots, g_{q}\right) \in \mathcal{H}:=$ $\left(L^{2}(0, T)\right)^{r} \times \mathbb{C}^{k q}$. The problem is to estimate the expression

$$
l(F)=\int_{0}^{T}\left(f(t), l_{0}(t)\right)_{r} d t+\sum_{i=1}^{q}\left(g_{i}, a_{i}\right)_{k}
$$

\footnotetext{
${ }^{1}$ Here and in what follows we assume that if a function $\varphi(t)$ is piecewise continuous then it is continuous from the left. If $\varphi(t)$ has a discontinuity of the first kind from the right at a certain point $t_{i}$, we denote by $\left.\Delta \varphi(t)\right|_{t=t_{i}}=\varphi\left(t_{i}^{+}\right)-\varphi\left(t_{i}\right)$ the jump of function $\varphi(t)$ at this point, $\varphi\left(t_{i}^{+}\right)=\lim _{t \rightarrow t_{i}+} \varphi(t)$.
} 
from observations of the form

$$
y_{j}^{i}(t)=H_{j}^{i}(t) x(t)+\xi_{j}^{i}(t), \quad t \in \Omega_{j}^{i}, \quad j=1, \ldots, M_{i}, \quad i=1, \ldots, q+1,
$$

in the class of estimates

$$
\widehat{l(F)}=\sum_{i=1}^{q+1} \sum_{j=1}^{M_{i}} \int_{\Omega_{j}^{i}}\left(y_{j}^{i}(t), u_{j}^{i}(t)\right)_{l} d t+c,
$$

linear with respect to observations (8); here $x(t)$ is the state of a system described by problem (5), (6), $l_{0} \in\left(L^{2}(0, T)\right)^{r}, a_{i} \in \mathbb{C}^{k}, H_{j}^{i}(t)$ are $l \times n$ matrices with the entries that are piecewise continuous functions on $\bar{\Omega}_{j}^{i}, u_{j}^{i}(t)$ are vector-functions belonging to $\left(L^{2}\left(\Omega_{j}^{i}\right)\right)^{l}, c \in \mathbb{C}$, and by $(\cdot, \cdot)_{m}$ we denote the inner product in $\mathbb{C}^{m}$.

We suppose that vector-function $f$ and vectors $g_{1}, \ldots, g_{q}$ are unknown and the element $F=$ $\left(f, g_{1}, \ldots, g_{q}\right)$ belongs to the set $G_{1}$, where

$$
\begin{aligned}
G_{1}=\left\{\tilde{F}:=\left(\tilde{f}, \tilde{g}_{1}, \ldots, \tilde{g}_{q}\right) \in \mathcal{H}: \tilde{f} \in\left(L^{2}(0, T)\right)^{r}, \tilde{g}_{i} \in \mathbb{C}^{k}, \sum_{i=1}^{q}\left(Q_{i}\left(\tilde{g}_{i}-g_{i}^{0}\right), \tilde{g}_{i}-g_{i}^{0}\right)_{k}\right. \\
\left.+\int_{0}^{T}\left(Q(t)\left(\tilde{f}(t)-f_{0}(t)\right), \tilde{f}(t)-f_{0}(t)\right)_{r} d t \leqslant 1\right\}
\end{aligned}
$$

$\xi:=\left(\xi_{1}^{1}(\cdot), \ldots, \xi_{M_{1}}^{1}(\cdot), \ldots, \xi_{1}^{q+1}(\cdot), \ldots, \xi_{M_{q+1}}^{q+1}(\cdot)\right) \in G_{2}$, where $\xi_{j}^{i}(\cdot)$ are observation errors in (8), that are realizations of random vector-functions $\xi_{j}^{i}(t)=\xi_{j}^{i}(\omega, t) \in \mathbb{C}^{l}$ and $G_{2}$ denotes the set of random elements $\tilde{\xi}:=\left(\tilde{\xi}_{1}^{1}(\cdot), \ldots, \tilde{\xi}_{M_{1}}^{1}(\cdot), \ldots, \tilde{\xi}_{1}^{q+1}(\cdot), \ldots, \tilde{\xi}_{M_{q+1}}^{q+1}(\cdot)\right)$, whose components have zero means, $\mathbb{E} \tilde{\xi}_{j}^{i}(\cdot)=0$, with Lebesgue square integrable second moments on $\Omega_{j}^{i}$, and unknown correlation matrices $\tilde{R}_{j}^{i}(t, s)=\mathbb{E} \tilde{\xi}_{j}^{i}(t)\left(\tilde{\xi}_{j}^{i}\right)^{*}(s)$ satisfying the condition

$$
\sum_{i=1}^{q+1} \sum_{j=1}^{M_{i}} \int_{\Omega_{j}^{i}} \operatorname{Tr}\left[D_{j}^{i}(t) \tilde{R}_{j}^{i}(t, t)\right] d t \leqslant 1,
$$

Here $f_{0}(t)$ is a prescribed vector-function such that $f_{0} \in\left(L^{2}(0, T)\right)^{r}, g_{1}^{0}, \ldots, g_{q}^{0} \in \mathbb{C}^{k}$ are prescribed vectors, $D_{j}^{i}(t)$ and $Q(t)$ are Hermitian $l \times l$ and $r \times r$-matrices with entries that are piecewise continuous functions on $\bar{\Omega}_{j}$ and $[0, T]$, respectively, such that $\int_{\Omega_{j}^{i}}\left(D_{j}^{i}(t) u(t), u(t)\right)_{l} d t \geqslant \alpha\|u\|_{\left(L^{2}\left(\Omega_{j}^{i}\right)\right)^{l}}$ and $\int_{0}^{T}(Q(t) v(t), v(t))_{r} d t \geqslant \beta\|v\|_{\left(L^{2}\left(\Omega_{j}^{i}\right)\right)^{r}}$ for all $u(\cdot) \in\left(L^{2}\left(\Omega_{j}^{i}\right)\right)^{l}$ and $v(\cdot) \in\left(L^{2}(0, T)\right)^{r}, \alpha, \beta=$ const $>0$, $Q_{i}, i=1, \ldots, q$ are Hermitian positive matrices with constant elements for which there exist their inverse matrices $\left(D_{j}^{i}\right)^{-1}(t), Q^{-1}(t)$, and $Q_{i}^{-1}$, $\operatorname{Tr} D:=\sum_{i=1}^{l} d_{i i}$ denotes the trace of the matrix $D=\left\{d_{i j}\right\}_{i, j=1}^{l}$.

Set $u:=\left(u_{1}^{1}(\cdot), \ldots, u_{M_{1}}^{1}(\cdot), \ldots, u_{1}^{q+1}(\cdot), \ldots, u_{M_{q+1}}^{q+1}(\cdot)\right) \in H$, where $H:=\left(L^{2}\left(\Omega_{1}^{1}\right)\right)^{l} \times \cdots \times$ $\left(L^{2}\left(\Omega_{M_{1}}^{1}\right)\right)^{l} \times \ldots \times\left(L^{2}\left(\Omega_{1}^{q+1}\right)\right)^{l} \times \ldots \times\left(L^{2}\left(\Omega_{M_{q+1}}^{q+1}\right)\right)^{l}$. Norm in space $H$ is defined by

$$
\|u\|_{H}=\left\{\sum_{i=1}^{q+1} \sum_{j=1}^{M_{i}}\left\|u_{j}^{i}(\cdot)\right\|_{\left(L^{2}\left(\Omega_{j}^{i}\right)\right)^{l}}\right\}^{1 / 2} .
$$

Definition 1. The estimate

$$
\widehat{\widehat{l(F)}}=\sum_{i=1}^{q+1} \sum_{j=1}^{M_{i}} \int_{\Omega_{j}^{i}}\left(y_{j}^{i}(t), \hat{u}_{j}^{i}(t)\right)_{l} d t+\hat{c},
$$

in which vector-functions $\hat{u}_{j}^{i}(\cdot)$, and a number $\hat{c}$ are determined from the condition 


$$
\inf _{u \in H, c \in \mathbb{C}} \sigma(u, c)=\sigma(\hat{u}, \hat{c})
$$

where

$$
\begin{gathered}
\sigma(u, c)=\sup _{\tilde{F} \in G_{1}, \tilde{\xi} \in G_{2}} \mathbb{E}|l(\tilde{F})-\widehat{l(\tilde{F})}|^{2}, \\
\widehat{l(\tilde{F})}=\sum_{i=1}^{q+1} \sum_{j=1}^{M_{i}} \int_{\Omega_{j}^{i}}\left(\tilde{y}_{j}^{i}(t), u_{j}^{i}(t)\right)_{l} d t+c, \\
\tilde{y}_{j}^{i}(t)=H_{j}^{i}(t) \tilde{x}(t)+\tilde{\xi}_{j}^{i}(t), \quad t \in \Omega_{j}^{i}, \quad j=1, \ldots, M_{i}, \quad i=1, \ldots, q+1,
\end{gathered}
$$

and $\tilde{x}(t)$ is the solution to the problem (5), (6) at $f(t)=\tilde{f}(t)$ and $g_{1}=\tilde{g}_{1}, \ldots g_{q}=\tilde{g}_{q}$, will be called the guaranteed (minimax) estimate of expression (7).

The quantity

$$
\sigma:=\{\sigma(\hat{u}, \hat{c})\}^{1 / 2}
$$

will be called the error of the guaranteed estimation of $l(F)$,

Thus, a guaranteed estimate is an estimate minimizing the maximal mean-square estimation error calculated for the worst-case realization of the perturbations.

\section{Representations for guaranteed estimates and estimation errors of $l(F)$}

In this section we deduce equations generated the guaranteed estimates.

For any fixed $u \in H$ introduce the vector-function $z(t ; u)$ as a unique solution to the problem

$$
\begin{gathered}
-\frac{d z(t ; u)}{d t}=A^{*}(t) z(t ; u)-\sum_{i=1}^{q+1} \sum_{j=1}^{M_{i}} \chi_{\Omega_{j}^{i}}(t)\left(H_{j}^{i}\right)^{*}(t) u_{j}^{i}(t) \quad \text { for a.e. } \quad t \in(0, T), \\
\left.\Delta z(t ; u)\right|_{t=t_{i}}=-\left(E+B_{i}^{*}\right)^{-1} B_{i}^{*} z\left(t_{i} ; u\right), \quad i=1, \ldots, q, \quad z(T ; u)=z(0 ; u),
\end{gathered}
$$

where $\chi_{\Omega}(t)=\left\{\begin{array}{ll}1 & \text { if } t \in \Omega, \\ 0 & \text { if } t \notin \Omega\end{array}\right.$ is a characteristic function of the set $\Omega$.

The unique solvability of this problem follows from condition (4) (see page 318) and from the results contained in [4] and [3] it also follows that an a priori estimate

$$
|z(t ; u)| \leqslant K \int_{t_{0}}^{T}\left|\sum_{i=1}^{q+1} \sum_{j=1}^{M_{i}} \chi_{\Omega_{j}^{i}}(s)\left(H_{j}^{i}\right)^{*}(s) u_{j}^{i}(s)\right| d s \quad \forall t \in[0, T]
$$

holds, where $K$ is a constant not depending on $u_{j}^{i}(\cdot)$.

Lemma 1. Finding the minimax estimate of functional $l(F)$ is equivalent to the problem of optimal control of the system (15), (16) with the cost function

$$
\begin{aligned}
I(u)=\int_{0}^{T}\left(Q^{-1}(t)\left(B^{*}(t) z(t ; u)+l_{0}(t)\right), B^{*}(t) z(t ; u)+l_{0}(t)\right)_{r} d t & \\
+ & +\sum_{i=1}^{q}\left(Q_{i}^{-1}\left(C_{i}^{*}\left(E+B_{i}^{*}\right)^{-1} z\left(t_{i} ; u\right)+a_{i}\right), C_{i}^{*}\left(E+B_{i}^{*}\right)^{-1} z\left(t_{i} ; u\right)+a_{i}\right)_{k} \\
& +\sum_{i=1}^{q+1} \sum_{j=1}^{M_{i}} \int_{\Omega_{j}^{i}}\left(\left(D_{j}^{i}\right)^{-1}(t) u_{j}^{i}(t), u_{j}^{i}(t)\right)_{l} d t \rightarrow \inf _{u \in H} .
\end{aligned}
$$

Mathematical Modeling and Computing, Vol. 8, No. 2, pp. 317-329 (2021) 
Proof. Let $\tilde{x}$ be a solution to problem (5), (6) at $f(t)=\tilde{f}(t), g_{i}=\tilde{g}_{i}, i=1, \ldots, q$. From (13) and (14), we obtain

$$
\begin{aligned}
\widehat{l(\tilde{F})} & =\sum_{i=1}^{q+1} \sum_{j=1}^{M_{i}} \int_{\Omega_{j}^{i}}\left(\tilde{y}_{j}^{i}(t), u_{j}^{i}(t)\right)_{l} d t+c \\
& =\sum_{i=1}^{q+1} \int_{t_{i-1}}^{t_{i}}\left(\tilde{x}(t), \sum_{j=1}^{M_{i}} \chi_{\Omega_{j}^{i}}(t)\left(H_{j}^{i}\right)^{*}(t) u_{j}^{i}(t)\right)_{n} d t+\sum_{i=1}^{q+1} \sum_{j=1}^{M_{i}} \int_{\Omega_{j}^{i}}\left(\tilde{\xi}_{j}^{i}(t), u_{j}(t)\right)_{l} d t+c
\end{aligned}
$$

Transform the first term in the right-hand side of this equality. Applying the integration by parts formula, we have

$$
\begin{gathered}
\sum_{i=1}^{q+1} \int_{t_{i-1}}^{t_{i}}\left(\tilde{x}(t), \sum_{j=1}^{M_{i}} \chi_{\Omega_{j}^{i}}(t)\left(H_{j}^{i}\right)^{*}(t) u_{j}^{i}(t)\right)_{n} d t=-\sum_{i=1}^{q+1} \int_{t_{i-1}}^{t_{i}}\left(\tilde{x}(t),-\frac{d z(t ; u)}{d t}-A^{*}(t) z(t ; u)\right)_{n} d t \\
=-\sum_{i=1}^{q+1}\left(\left(\tilde{x}\left(t_{i-1}^{+}\right), z\left(t_{i-1}^{+} ; u\right)\right)_{n}-\left(\tilde{x}\left(t_{i}\right), z\left(t_{i} ; u\right)\right)_{n}\right)-\sum_{i=1}^{q+1} \int_{t_{i-1}}^{t_{i}}\left(\frac{d \tilde{x}(t)}{d t}-A(t) \tilde{x}(t), z(t ; u)\right)_{n} d t \\
=-\sum_{i=1}^{q}\left(C_{i} \tilde{g}_{i},\left(E+B_{i}^{*}\right)^{-1} z\left(t_{i}\right)\right)_{n}-\sum_{i=1}^{q+1} \int_{t_{i-1}}^{t_{i}}(B(t) \tilde{f}(t), z(t ; u))_{n} d t
\end{gathered}
$$

Here we have used the fact that

$$
\begin{gathered}
\sum_{i=1}^{q+1}\left(\left(\tilde{x}\left(t_{i-1}^{+}\right), z\left(t_{i-1}^{+} ; u\right)\right)_{n}-\left(\tilde{x}\left(t_{i}\right), z\left(t_{i} ; u\right)\right)_{n}\right)=\sum_{i=1}^{q}\left(\left(\tilde{x}\left(t_{i}^{+}\right), z\left(t_{i}^{+} ; u\right)\right)_{n}-\left(\tilde{x}\left(t_{i}\right), z\left(t_{i} ; u\right)\right)_{n}\right) \\
=\sum_{i=1}^{q}\left(\left(\left(E+B_{i}\right) \tilde{x}\left(t_{i}\right)+C_{i} \tilde{g}_{i},\left(E-\left(E+B_{i}^{*}\right)^{-1} B_{i}^{*}\right) z\left(t_{i} ; u\right)\right)_{n}-\left(\tilde{x}\left(t_{i}\right), z\left(t_{i} ; u\right)\right)_{n}\right) \\
=\sum_{i=1}^{q}\left(\left(\left(E+B_{i}\right) \tilde{x}\left(t_{i}\right),\left(E-\left(E+B_{i}^{*}\right)^{-1} B_{i}^{*}\right) z\left(t_{i} ; u\right)\right)_{n}-\left(\tilde{x}\left(t_{i}\right), z\left(t_{i} ; u\right)\right)_{n}\right) \\
+\sum_{i=1}^{q}\left(C_{i} \tilde{g}_{i},\left(E-\left(E+B_{i}^{*}\right)^{-1} B_{i}^{*}\right) z\left(t_{i} ; u\right)\right)_{n} \\
=\sum_{i=1}^{q}\left(\left(\left(E+B_{i}\right) \tilde{x}\left(t_{i}\right),\left(E+B_{i}^{*}\right)^{-1} z\left(t_{i} ; u\right)\right)_{n}-\left(\tilde{x}\left(t_{i}\right), z\left(t_{i} ; u\right)\right)_{n}\right)+\sum_{i=1}^{q}\left(C_{i} \tilde{g}_{i},\left(E+B_{i}^{*}\right)^{-1} z\left(t_{i} ; u\right)\right)_{n} \\
=\sum_{i=1}^{q}\left(\tilde{g}_{i}, C_{i}^{*}\left(E+B_{i}^{*}\right)^{-1} z\left(t_{i} ; u\right)\right)_{k} .
\end{gathered}
$$

Since

$$
l(\tilde{F})=\int_{0}^{T}\left(\tilde{f}(t), l_{0}(t)\right)_{r} d t+\sum_{i=1}^{q}\left(\tilde{g}_{i}, a_{i}\right)_{k}
$$

we get

$$
\begin{aligned}
l(\tilde{F})-\widehat{l(\tilde{F})}=\int_{0}^{T}\left(\tilde{f}(t), l_{0}(t)+B^{*}(t) z(t ; u)\right)_{r} d t+\sum_{i=1}^{q}\left(\tilde{g}_{i}, a_{i}\right. & \left.+C_{i}^{*}\left(E+B_{i}^{*}\right)^{-1} z\left(t_{i} ; u\right)\right)_{k} \\
& -\sum_{i=1}^{q+1} \sum_{j=1}^{M_{i}} \int_{\Omega_{j}^{i}}\left(\tilde{\xi}_{j}^{i}(t), u_{j}(t)\right)_{l} d t-c
\end{aligned}
$$

Mathematical Modeling and Computing, Vol. 8, No. 2, pp. 317-329 (2021) 
The latter equality yields

$$
\mathbb{E}[l(\tilde{F})-\widehat{l(\tilde{F})}]=\int_{0}^{T}\left(\tilde{f}(t), l_{0}(t)+B^{*}(t) z(t ; u)\right)_{r} d t+\sum_{i=1}^{q}\left(\tilde{g}_{i}, a_{i}+C_{i}^{*}\left(E+B_{i}^{*}\right)^{-1} z\left(t_{i} ; u\right)\right)_{k}-c .
$$

From the equalities (20) and (21) we find

$$
\begin{array}{r}
\mathbb{E}|l(\tilde{F})-\widehat{l(\tilde{F})}|^{2}=\left|\int_{0}^{T}\left(\tilde{f}(t), l_{0}(t)+B^{*}(t) z(t ; u)\right)_{r} d t+\sum_{i=1}^{q}\left(\tilde{g}_{i}, a_{i}+C_{i}^{*}\left(E+B_{i}^{*}\right)^{-1} z\left(t_{i} ; u\right)\right)_{k}-c\right|^{2} \\
\quad+\mathbb{E}\left|\sum_{i=1}^{q+1} \sum_{j=1}^{M_{i}} \int_{\Omega_{j}^{i}}\left(\tilde{\xi}_{j}^{i}(t), u_{j}(t)\right)_{l} d t\right|^{2} \\
\left.=\mid \int_{0}^{T}\left(\tilde{f}(t)-f_{0}(t), l_{0}(t)+B^{*}(t) z(t ; u)\right)_{r} d t\right)_{r} d t+\sum_{i=1}^{q}\left(\tilde{g}_{i}-g_{i}^{0}, a_{i}+C_{i}^{*}\left(E+B_{i}^{*}\right)^{-1} z\left(t_{i} ; u\right)\right)_{k} \\
\left.+\int_{0}^{T}\left(f_{0}(t), l_{0}(t)+B^{*}(t) z(t ; u)\right)_{r} d t\right)_{r} d t+\sum_{i=1}^{q}\left(g_{i}^{0}, a_{i}+C_{i}^{*}\left(E+B_{i}^{*}\right)^{-1} z\left(t_{i} ; u\right)\right)_{k}-\left.c\right|^{2} \\
+\mathbb{E}\left|\sum_{i=1}^{q+1} \sum_{j=1}^{M_{i}} \int_{\Omega_{j}^{i}}\left(\tilde{\xi}_{j}^{i}(t), u_{j}(t)\right)_{l} d t\right|^{2} .
\end{array}
$$

Thus,

$$
\begin{aligned}
& \inf _{c \in \mathbb{C}} \sigma(u, c)=\inf _{c \in \mathbb{C}} \sup _{\tilde{F} \in G_{1}, \tilde{\xi} \in G_{2}} \mathbb{E}|l(\tilde{F})-\widehat{l(\tilde{F})}|^{2} \\
& \left.=\inf _{c \in \mathbb{C}} \sup _{\tilde{F} \in G_{1}} \mid \int_{0}^{T}\left(\tilde{f}(t)-f_{0}(t), l_{0}(t)+B^{*}(t) z(t ; u)\right)_{r} d t\right)_{r} d t+\sum_{i=1}^{q}\left(\tilde{g}_{i}-g_{i}^{0}, a_{i}+C_{i}^{*}\left(E+B_{i}^{*}\right)^{-1} z\left(t_{i} ; u\right)\right)_{k} \\
& \left.\quad+\int_{0}^{T}\left(f_{0}(t), l_{0}(t)+B^{*}(t) z(t ; u)\right)_{r} d t\right)_{r} d t+\sum_{i=1}^{q}\left(g_{i}^{0}, a_{i}+C_{i}^{*}\left(E+B_{i}^{*}\right)^{-1} z\left(t_{i} ; u\right)\right)_{k}-\left.c\right|^{2} \\
& \quad+\sup _{\tilde{\xi} \in G_{2}} \mathbb{E}\left|\sum_{i=1}^{q+1} \sum_{j=1}^{M_{i}} \int_{\Omega_{j}^{i}}\left(\tilde{\xi}_{j}^{i}(t), u_{j}(t)\right)_{l} d t\right|^{2} \cdot \quad(22)
\end{aligned}
$$

Set

$$
\begin{gathered}
\left.y:=\int_{0}^{T}\left(\tilde{f}(t)-f_{0}(t), l_{0}(t)+B^{*}(t) z(t ; u)\right)_{r} d t\right)_{r} d t+\sum_{i=1}^{q}\left(\tilde{g}_{i}-g_{i}^{0}, a_{i}+C_{i}^{*}\left(E+B_{i}^{*}\right)^{-1} z\left(t_{i} ; u\right)\right)_{k}, \\
\left.d=c-\int_{0}^{T}\left(f_{0}(t), l_{0}(t)+B^{*}(t) z(t ; u)\right)_{r} d t\right)_{r} d t-\sum_{i=1}^{q}\left(g_{i}^{0}, a_{i}+C_{i}^{*}\left(E+B_{i}^{*}\right)^{-1} z\left(t_{i} ; u\right)\right)_{k} .
\end{gathered}
$$

Then generalized Cauchy-Schwarz inequaity and (10) imply

$$
\begin{aligned}
|y| \leqslant\left[\int_{0}^{T}(\right. & \left.Q^{-1}(t)\left(l_{0}(t)+B^{*}(t) z(t ; u)\right), l_{0}(t)+B^{*}(t) z(t ; u)\right)_{r} d t \\
& \left.+\sum_{i=1}^{q}\left(Q_{i}^{-1}\left(a_{i}+C_{i}^{*}\left(E+B_{i}^{*}\right)^{-1} z\left(t_{i}\right)\right), a_{i}+C_{i}^{*}\left(E+B_{i}^{*}\right)^{-1} z\left(t_{i} ; u\right)\right)_{k}\right]^{1 / 2} \\
\times & {\left[\sum_{i=1}^{q}\left(Q_{i}\left(\tilde{g}_{i}-g_{i}^{0}\right), \tilde{g}_{i}-g_{i}^{0}\right)_{k}+\int_{0}^{T}\left(Q(t)\left(\tilde{f}(t)-f_{0}(t)\right), \tilde{f}(t)-f_{0}(t)\right)_{r} d t\right]^{1 / 2} }
\end{aligned}
$$

Mathematical Modeling and Computing, Vol. 8, No. 2, pp. 317-329 (2021) 


$$
\begin{aligned}
\leqslant & {\left[\int_{0}^{T}\left(Q^{-1}(t)\left(l_{0}(t)+B^{*}(t) z(t ; u)\right), l_{0}(t)+B^{*}(t) z(t ; u)\right)_{r} d t\right.} \\
& \left.+\sum_{i=1}^{q}\left(Q_{i}^{-1}\left(a_{i}+C_{i}^{*}\left(E+B_{i}^{*}\right)^{-1} z\left(t_{i}\right)\right), a_{i}+C_{i}^{*}\left(E+B_{i}^{*}\right)^{-1} z\left(t_{i} ; u\right)\right)_{k}\right]^{1 / 2}=: J .
\end{aligned}
$$

The direct substitution shows that last inequality is transformed to an equality at $\check{F}:=\left(\check{f}, \check{g}_{1}, \ldots, \check{g}_{q}\right) \in$ $G_{1}$, where

$$
\begin{gathered}
\check{f}(t)=f_{0}(t) \pm \frac{1}{J}\left(Q_{i}^{1}\right)^{-1}(t)\left(l_{0}(t)+B^{*}(t) z(t ; u)\right), \\
\check{g}_{i}=g_{i}^{0} \pm \frac{1}{J} Q_{i}^{-1}\left(a_{i}+C_{i}^{*}\left(E+B_{i}^{*}\right)^{-1} z\left(t_{i} ; u\right)\right),
\end{gathered}
$$

Taking into account the equality

$$
\inf _{d \in \mathbb{C}} \sup _{|y| \leqslant J}|y-d|^{2}=J^{2},
$$

we find

$$
\begin{gathered}
\left.\inf _{c \in \mathbb{C}} \sup _{\tilde{F} \in G_{1}} \mid \int_{0}^{T}\left(\tilde{f}(t)-f_{0}(t), l_{0}(t)+B^{*}(t) z(t ; u)\right)_{r} d t\right)_{r} d t+\sum_{i=1}^{q}\left(\tilde{g}_{i}-g_{i}^{0}, a_{i}+C_{i}^{*}\left(E+B_{i}^{*}\right)^{-1} z\left(t_{i} ; u\right)\right)_{k} \\
\left.+\int_{0}^{T}\left(f_{0}(t), l_{0}(t)+B^{*}(t) z(t ; u)\right)_{r} d t\right)_{r} d t+\sum_{i=1}^{q}\left(g_{i}^{0}, a_{i}+C_{i}^{*}\left(E+B_{i}^{*}\right)^{-1} z\left(t_{i} ; u\right)\right)_{k}-\left.c\right|^{2} \\
=J^{2}=\int_{0}^{T}\left(Q^{-1}(t)\left(l_{0}(t)+B^{*}(t) z(t ; u)\right), l_{0}(t)+B^{*}(t) z(t ; u)\right)_{r} d t \\
+\sum_{i=1}^{q}\left(Q_{i}^{-1}\left(a_{i}+C_{i}^{*}\left(E+B_{i}^{*}\right)^{-1} z\left(t_{i}\right)\right), a_{i}+C_{i}^{*}\left(E+B_{i}^{*}\right)^{-1} z\left(t_{i} ; u\right)\right)_{k}, \quad
\end{gathered}
$$

where the infimum over $c$ is attained at

$$
\left.c=\int_{0}^{T}\left(f_{0}(t), l_{0}(t)+B^{*}(t) z(t ; u)\right)_{r} d t\right)_{r} d t+\sum_{i=1}^{q}\left(g_{i}^{0}, a_{i}+C_{i}^{*}\left(E+B_{i}^{*}\right)^{-1} z\left(t_{i} ; u\right)\right)_{k} .
$$

The last term on the right-hand side of $(22)$ is calculated analogously. As a result, we get

$$
\sup _{\tilde{\xi} \in G_{2}} \mathbb{E}\left|\sum_{i=1}^{q+1} \sum_{j=1}^{M_{i}} \int_{\Omega_{j}^{i}}\left(\tilde{\xi}_{j}^{i}(t), u_{j}(t)\right)_{l} d t\right|^{2}=\sum_{i=1}^{q+1} \sum_{j=1}^{M_{i}} \int_{\Omega_{j}^{i}}\left(\left(D_{j}^{i}\right)^{-1}(t) u_{j}^{i}(t), u_{j}^{i}(t)\right)_{l} d t .
$$

The statement of the lemma follows now from (22), (23), (24) and (25). The proof is complete.

Further in the proof of Theorem 1 stated below, it will be shown that solving the optimal control problem (15)-(18) is reduced to solving some system of impulsive periodic differential equations.

Theorem 1. The minimax estimate $\widehat{\widehat{l(F)}}$ of expression $l(F)$ has the form

$$
\widehat{\widehat{l(F)}}=\sum_{i=1}^{q+1} \sum_{j=1}^{M_{i}} \int_{\Omega_{j}^{i}}\left(y_{j}^{i}(t), \hat{u}_{j}^{i}(t)\right)_{l} d t+\hat{c}=l(\hat{F})
$$

where

$$
\begin{gathered}
\hat{u}_{j}^{i}(t)=D_{j}^{i}(t) H_{j}^{i}(t) p(t), \quad i=1, \ldots, q+1, \quad j=1, \ldots, M_{i}, \\
\left.c=\int_{0}^{T}\left(f_{0}(t), l_{0}(t)+B^{*}(t) \hat{z}(t)\right)_{r} d t\right)_{r} d t+\sum_{i=1}^{q}\left(g_{i}^{0}, a_{i}+C_{i}^{*}\left(E+B_{i}^{*}\right)^{-1} \hat{z}\left(t_{i}\right)\right)_{k},
\end{gathered}
$$

$\hat{F}:=\left(\hat{f}, \hat{g}_{1}, \ldots, \hat{g}_{q}\right)$ with 


$$
\hat{f}(t)=f_{0}(t)+Q^{-1}(t) B^{*}(t) \hat{p}(t), \quad \hat{g}_{i}=g_{i}^{0}+Q_{i}^{-1} C_{i}^{*}\left(E+B_{i}^{*}\right)^{-1} \hat{p}\left(t_{i}\right), \quad i=1 \ldots, q,
$$

and vector-functions $p(t), \hat{z}(t)$, and $\hat{p}(t)$ are determined from the solution of the systems of equations

$$
\begin{gathered}
-\frac{d \hat{z}(t)}{d t}=A^{*}(t) \hat{z}(t)-\sum_{i=1}^{q+1} \sum_{j=1}^{M_{i}} \chi_{\Omega_{j}^{i}}(t)\left(H_{j}^{i}\right)^{*}(t) D_{j}^{i}(t) H_{j}^{i}(t) p(t) \quad \text { for a.e. } \quad t \in(0, T), \\
\left.\Delta \hat{z}(t)\right|_{t=t_{i}}=-\left(E+B_{i}^{*}\right)^{-1} B_{i}^{*} \hat{z}\left(t_{i}\right), \quad i=1, \ldots, q, \quad \hat{z}(T)=\hat{z}(0), \\
\frac{d p(t)}{d t}=A(t) p(t)+B(t) Q^{-1}(t)\left(B^{*} \hat{z}(t)+l_{0}(t)\right) \quad \text { for a.e. } \quad t \in(0, T), \\
\left.\Delta p(t)\right|_{t=t_{i}}=B_{i} p\left(t_{i}\right)+C_{i} Q_{i}^{-1}\left(C_{i}^{*}\left(E+B_{i}^{*}\right)^{-1} \hat{z}\left(t_{i}\right)+a_{i}\right), \quad i=1, \ldots, q, \quad p(0)=p(T)
\end{gathered}
$$

and

$$
\begin{gathered}
-\frac{d \hat{p}(t)}{d t}=A^{*}(t) \hat{p}(t)-\sum_{i=1}^{q+1} \sum_{j=1}^{M_{i}} \chi_{\Omega_{j}^{i}}(t)\left(H_{j}^{i}\right)^{*}(t) D_{j}^{i}(t)\left[H_{j}^{i}(t) \hat{x}(t)-y_{j}^{i}(t)\right] \quad \text { for a.e. } \quad t \in(0, T), \\
\left.\Delta \hat{p}(t)\right|_{t=t_{i}}=-\left(E+B_{i}^{*}\right)^{-1} B_{i}^{*} \hat{p}\left(t_{i}\right), \quad i=1, \ldots, q, \quad \hat{p}(T)=\hat{p}(0), \\
\frac{d \hat{x}(t)}{d t}=A(t) \hat{x}(t)+B(t)\left(Q^{-1}(t) B^{*}(t) \hat{p}(t)+f_{0}(t)\right) \quad \text { for a.e. } \quad t \in(0, T), \\
\left.\Delta \hat{x}(t)\right|_{t=t_{i}}=B_{i} \hat{x}\left(t_{i}\right)+C_{i} Q_{i}^{-1}\left(C_{i}^{*}\left(E+B_{i}^{*}\right)^{-1} \hat{p}\left(t_{i}\right)+g_{i}\right), \quad i=1, \ldots, q, \quad \hat{x}(0)=\hat{x}(T),
\end{gathered}
$$

respectively. Problems (29)-(32) and (33)-(36) are uniquely solvable. Equations (33)-(36) are fulfilled with probability 1.

The minimax estimation error $\sigma$ is determined by the formula

$$
\sigma=[l(\hat{P})]^{1 / 2},
$$

where

$$
\hat{P}=\left(Q^{-1}(\cdot)\left(l_{0}(\cdot)+B^{*}(\cdot) \hat{z}(\cdot)\right), Q_{1}^{-1}\left(C_{1}^{*}\left(E+B_{1}^{*}\right)^{-1} \hat{z}\left(t_{1}\right)+a_{1}, \ldots, Q_{q}^{-1}\left(C_{q}^{*}\left(E+B_{q}^{*}\right)^{-1} \hat{z}\left(t_{q}\right)+a_{q}\right) .\right.\right.
$$

Proof. It is not difficult to verify, using the inequality (17), that $I(u)$ is a weak lower semicontinuous strictly convex functional on $H$. Therefore, since

$$
I(u) \geqslant \sum_{i=1}^{q+1} \sum_{j=1}^{M_{i}} \int_{\Omega_{j}^{i}}\left(\left(D_{j}^{i}\right)^{-1}(t) u_{j}^{i}(t), u_{j}^{i}(t)\right)_{l} d t \geqslant c\|u\|_{H}^{2} \quad \forall u \in H, \quad \mathrm{c}=\mathrm{const},
$$

then, by Theorems 13.2 and 13.4 (see [6]), there exists one and only one element $\hat{u} \in H$ such that $I(\hat{u})=\inf _{u \in H} I(u)$. Hence, for any fixed $v \in H$ and $\tau \in \mathbb{R}$ the functions $s_{1}(\tau):=I(\hat{u}+\tau v)$ and $s_{2}(\tau):=I(\hat{u}+i \tau v)$ reach their minimums at a unique point $\tau=0$, so that

$$
\left.\frac{1}{2} \frac{d}{d \tau} I(\hat{u}+\tau v)\right|_{\tau=0}=0 \quad \text { and }\left.\quad \frac{1}{2} \frac{d}{d \tau} I(\hat{u}+i \tau v)\right|_{\tau=0}=0
$$

where $i=\sqrt{-1}$. Since $z(t ; \hat{u}+\tau v)=z(t ; \hat{u})+\tau z(t ; v)$ and $z(t ; \hat{u}+i \tau v)=z(t ; \hat{u})+i \tau z(t ; v)$, from $(18)$ and (38), we obtain

$$
0=\int_{0}^{T}\left(Q^{-1}(t)\left(B^{*}(t) z(t ; \hat{u})+l_{0}(t)\right), B^{*}(t) z(t ; v)\right)_{r} d t
$$

Mathematical Modeling and Computing, Vol. 8, No. 2, pp. 317-329 (2021) 


$$
\begin{aligned}
+\sum_{i=1}^{q}\left(Q _ { i } ^ { - 1 } \left(C_{i}^{*}\left(E+B_{i}^{*}\right)^{-1} z\left(t_{i} ; \hat{u}\right)+\right.\right. & \left.\left.a_{i}\right), C_{i}^{*}\left(E+B_{i}^{*}\right)^{-1} z\left(t_{i} ; v\right)\right)_{k} \\
& +\sum_{i=1}^{q+1} \sum_{j=1}^{M_{i}} \int_{\Omega_{j}^{i}}\left(\left(D_{j}^{i}\right)^{-1}(t) \hat{u}_{j}^{i}(t), v_{j}^{i}(t)\right)_{l} d t
\end{aligned}
$$

Let $p(t)$ be a solution of the problem

$$
\begin{gathered}
\frac{d p(t)}{d t}=A(t) p(t)+B(t) Q^{-1}(t)\left(B^{*} z(t ; \hat{u})+l_{0}(t)\right) \quad \text { for a.e. } \quad t \in(0, T), \\
\left.\Delta p(t)\right|_{t=t_{i}}=B_{i} p\left(t_{i}\right)+C_{i} Q_{i}^{-1}\left(C_{i}^{*}\left(E+B_{i}^{*}\right)^{-1} \hat{z}\left(t_{i} ; \hat{u}\right)+a_{i}\right), \quad i=1, \ldots, q, \quad p(0)=p(T) .
\end{gathered}
$$

Taking this into account, transform the first summand in the right-hand side of (39). We have

$$
\begin{gathered}
\int_{0}^{T}\left(Q^{-1}(t)\left(B^{*}(t) z(t ; \hat{u})+l_{0}(t)\right), B^{*}(t) z(t ; v)\right)_{r} d t \\
=\sum_{i=1}^{q+1} \int_{t_{i-1}}^{t_{i}}\left(\frac{d p(t)}{d t}-A(t) p(t), z(t ; v)\right)_{n} d t \\
=\sum_{i=1}^{q+1}\left(\left(p\left(t_{i}\right), z\left(t_{i} ; v\right)\right)_{n}-\left(p\left(t_{i-1}^{+}\right), z\left(t_{i-1}^{+} ; v\right)\right)_{n}\right)-\sum_{i=1}^{q+1} \int_{t_{i-1}}^{t_{i}}\left(p(t), \frac{d z(t ; v)}{d t}+A^{*}(t) z(t ; v)\right)_{n} d t \\
=-\sum_{i=1}^{q}\left(Q_{i}^{-1}\left(C_{i}^{*}\left(E+B_{i}^{*}\right)^{-1} z\left(t_{i} ; \hat{u}\right)+a_{i}\right), C_{i}^{*}\left(E+B_{i}^{*}\right)^{-1} z\left(t_{i} ; v\right)\right)_{k} \\
-\int_{0}^{T}\left(p(t), \sum_{i=1}^{q+1} \sum_{j=1}^{M_{i}} \chi_{\Omega_{j}^{i}}(t)\left(H_{j}^{i}\right)^{*}(t) v_{j}^{i}(t)\right)_{n} d t
\end{gathered}
$$

From (39), (40) we find

$$
\sum_{i=1}^{q+1} \sum_{j=1}^{M_{i}} \int_{\Omega_{j}^{i}}\left(\left(D_{j}^{i}\right)^{-1}(t) \hat{u}_{j}^{i}(t), v_{j}^{i}(t)\right)_{l} d t=\sum_{i=1}^{q+1} \sum_{j=1}^{M_{i}} \int_{\Omega_{j}^{i}}\left(p(t),\left(H_{j}^{i}\right)^{*}(t) v_{j}^{i}(t)\right)_{n} d t
$$

for any $v:=\left(v_{1}^{1}(\cdot), \ldots, v_{M_{1}}^{1}(\cdot), \ldots, v_{1}^{q+1}(\cdot), \ldots, v_{M_{q+1}}^{q+1}(\cdot)\right) \in H$, whence $\hat{u}_{j}^{i}(t), i=1, \ldots, q+1, j=$ $1, \ldots, M_{i}$ are defined by (26). Setting $u=\hat{u}$ in $(24),(15)$, and (16) and denoting $\hat{z}(t)=z(t ; \hat{u})$, we see that $\hat{z}(t)$ and $p(t)$ satisfy system $(29)-(32)$; the unique solvability of this system follows from the fact that functional $I(u)$ has one minimum point $\hat{u}$.

Now let us establish that $\sigma=[l(\hat{P})]^{1 / 2}$. Substituting expression (26) into (18), we obtain

$$
\begin{aligned}
\sigma^{2}=I(\hat{u})=\int_{0}^{T} & \left(Q^{-1}(t)\left(B^{*}(t) \hat{z}(t)+l_{0}(t)\right), B^{*}(t) \hat{z}(t)+l_{0}(t)\right)_{r} d t \\
& +\sum_{i=1}^{q}\left(Q_{i}^{-1}\left(C_{i}^{*}\left(E+B_{i}^{*}\right)^{-1} \hat{z}\left(t_{i}\right)+a_{i}\right), C_{i}^{*}\left(E+B_{i}^{*}\right)^{-1} \hat{z}\left(t_{i}\right)+a_{i}\right)_{k} \\
& +\sum_{i=1}^{q+1} \sum_{j=1}^{M_{i}} \int_{\Omega_{j}^{i}}\left(H_{j}^{i}(t) p(t), D_{j}^{i}(t) H_{j}^{i}(t) p(t)\right)_{l} d t
\end{aligned}
$$

However,

$$
\int_{0}^{T}\left(Q^{-1}(t)\left(B^{*}(t) \hat{z}(t)+l_{0}(t)\right), B^{*}(t) \hat{z}(t)\right)_{r} d t=\sum_{i=1}^{q+1} \int_{t_{i-1}}^{t_{i}}\left(\frac{d p(t)}{d t}-A(t) p(t), \hat{z}(t)\right)_{n} d t
$$

Mathematical Modeling and Computing, Vol. 8, No. 2, pp. 317-329 (2021) 


$$
\begin{gathered}
=\sum_{i=1}^{q+1}\left(\left(p\left(t_{i}\right), \hat{z}\left(t_{i}\right)\right)_{n}-\left(p\left(t_{i-1}^{+}\right), \hat{z}\left(t_{i-1}^{+}\right)\right)_{n}\right)-\sum_{i=1}^{q+1} \int_{t_{i-1}}^{t_{i}}\left(p(t), \frac{d \hat{z}(t)}{d t}+A^{*}(t) \hat{z}(t)\right)_{n} d t \\
=-\sum_{i=1}^{q}\left(Q_{i}^{-1}\left(C_{i}^{*}\left(E+B_{i}^{*}\right)^{-1} \hat{z}\left(t_{i}\right)+a_{i}\right), C_{i}^{*}\left(E+B_{i}^{*}\right)^{-1} \hat{z}\left(t_{i}\right)\right)_{k}-\sum_{i=1}^{q+1} \sum_{j=1}^{M_{i}} \int_{\Omega_{j}^{i}}\left(H_{j}^{i}(t) p(t), D_{j}^{i}(t) H_{j}^{i}(t) p(t)\right)_{l} d t .
\end{gathered}
$$

From here and from (41) it follows (37).

The representation

$$
\widehat{\widehat{l(F)}}=l(\hat{F})
$$

can be proved in much the same way as the representation

$$
\widehat{\widehat{l(F)}}=\sum_{i=1}^{q+1} \sum_{j=1}^{M_{i}} \int_{\Omega_{j}^{i}}\left(y_{j}^{i}(t), \hat{u}_{j}^{i}(t)\right)_{l} d t+\hat{c} .
$$

This completes the proof.

Remark 1. In the representation $\widehat{\widehat{l(F)}}=l(\hat{F})$ of the guaranteed mean square estimate of $l(F)$, where $F:=\left(f, g_{1}, \ldots, g_{q}\right), \hat{F}:=\left(\hat{f}, \hat{g}_{1}, \ldots, \hat{g}_{q}\right)$ with

$$
\hat{f}(t)=f_{0}(t)+Q^{-1}(t) B^{*}(t) \hat{p}(t), \quad \hat{g}_{i}=g_{i}^{0}+Q_{i}^{-1} C_{i}^{*}\left(E+B_{i}^{*}\right)^{-1} \hat{p}\left(t_{i}\right), \quad i=1 \ldots, q,
$$

vector-function $\hat{f}(t)$ and vectors $\hat{g}_{i}$ do not depend on a specific form of functional $l$.

\section{Optimal estimation problem of unknown data}

Now consider the problem of finding optimal estimate of the vector $g=L F$ among estimates of the form

$$
\hat{g}=\sum_{i=1}^{q+1} \sum_{j=1}^{M_{i}} U_{j}^{i} y_{j}^{i}(\cdot)+C ;
$$

here $y_{j}^{i}(\cdot)$ are observations $(8), L$ is a linear continuous operator acting from the space $\mathcal{H}$ into a separable complex Hilbert space $V$ with inner product $(\cdot, \cdot)$ and norm $\|\cdot\|, U_{j}^{i}$ are linear continuous operators acting from $\left(L^{2}\left(\Omega_{j}^{i}\right)\right)^{l}$ to $V, C \in V$.

Let $\left\{e_{1}, e_{2}, \ldots\right\}$ be an orthonormal basis of $V$. Denote by $\sigma_{1}(U, C)$ and $\sigma_{2}(U, C)$ the quantities defined by

$$
\sigma_{1}(U, C)=\sup _{G_{1}, G_{2}} \mathbb{E}\|g-\hat{g}\|^{2}
$$

and

$$
\sigma_{2}(U, C)=\sum_{k=1}^{\infty} \sup _{G_{1}, G_{2}} \mathbb{E}\left|\left(g-\hat{g}, e_{k}\right)\right|^{2}
$$

respectively, where $U:=\left(U_{1}^{1}, \ldots, U_{M_{1}}^{1}, \ldots, U_{1}^{q+1}, \ldots, U_{M_{q+1}}^{q+1}\right), G_{1}$ and $G_{2}$ are defined on page 319 .

Definition 2. The estimates $\hat{\hat{g}}_{1}$ and $\hat{\hat{g}}_{2}$ which are determined from the condition ${ }^{2}$

$$
\hat{\hat{g}}_{i} \in \underset{\hat{g} \in \mathcal{L}}{\operatorname{Argmin}} \sigma_{i}(U, C)
$$

\footnotetext{
${ }^{2}$ Notice, that Definition 1 is a special case of the Definition 2.
} 
are called the guaranteed and optimal estimate of $g$, respectively, where by $\mathcal{L}$ we denote the set of all estimates of the form (43).

Parseval's formula implies that the following inequality holds

$$
\sigma_{1}(U, C) \leqslant \sigma_{2}(U, C)
$$

The equality sign in this inequality holds if $V=\mathbb{R}$.

Lemma 2. Suppose that for an arbitrary vector $e \in V$ there holds the equality

$$
\inf _{(g, e)} \sup _{G_{1}, G_{2}} \mathbb{E}|(g, e)-\widehat{(g, e)}|^{2}=\sup _{G_{1}, G_{2}} \mathbb{E}|(g, e)-\widehat{\widehat{(g, e)}}|^{2},
$$

where $\widehat{\widehat{(g, e)}}=(\hat{\hat{g}}, e), \hat{\hat{g}}$ does not depend on vector $e$, and $\widehat{(g, e)}$ is a linear estimate of inner product $(g, e)$. Then vector $\hat{\hat{g}}$ is the optimal estimate of vector $g$.

Proof. Notice that

$$
\begin{aligned}
\inf _{\hat{g} \in \mathcal{L}} \sigma_{2}(U, C) & =\inf _{\hat{g} \in \mathcal{L}} \sum_{k=1}^{\infty} \sup _{G_{1}, G_{2}} \mathbb{E}\left|\left(g-\hat{g}, e_{k}\right)\right|^{2} \geqslant \sum_{k=1}^{\infty} \frac{\inf }{\left(g, e_{k}\right)} \sup _{G_{1}, G_{2}} \mathbb{E}\left|\left(g, e_{k}\right)-\widehat{\left(g, e_{k}\right)}\right|^{2} \\
& =\sup _{G_{1}, G_{2}} \mathbb{E}\left|\left(g, e_{k}\right)-\widehat{\widehat{\left(g, e_{k}\right)}}\right|^{2}=\sum_{k=1}^{\infty} \sup _{G_{1}, G_{2}} \mathbb{E}\left|\left(g-\hat{\hat{g}}, e_{k}\right)\right|^{2}
\end{aligned}
$$

and the lower bound is attained at $\hat{g}=\hat{\hat{g}}$. This completes the proof.

Further we will obtain the optimal estimate of element $g=L F$ using this lemma. Note first that for any $e \in V$, we have

$$
\begin{aligned}
(g, e)-(\hat{g}, e) & =(L F, e)-\left(\sum_{i=1}^{q+1} \sum_{j=1}^{M_{i}} U_{j}^{i} y_{j}^{i}(\cdot)+C, e\right) \\
& =\left(F, L^{*} e\right)_{\mathcal{H}}-\sum_{i=1}^{q+1} \sum_{j=1}^{M_{i}} \int_{\Omega_{j}^{i}}\left(y_{j}^{i}(t),\left(U_{j}^{i}\right)^{*} e(t)\right)_{n} d t-(C, e) \\
& =l(F)-\widehat{l(F)},
\end{aligned}
$$

where $L^{*}$ and $\left(U_{j}^{i}\right)^{*}$ denote the adjoint operators of $L$ and $U_{j}^{i}$, respectively,

$$
l(F):=\left(F, L^{*} e\right)_{\mathcal{H}}=\int_{0}^{T}\left(f(t), l_{0}(t)\right)_{r} d t+\sum_{i=1}^{q}\left(g_{i}, a_{i}\right)_{k}
$$

with some $l_{0} \in\left(L^{2}(0, T)^{r}\right.$, and $a_{i} \in \mathbb{C}^{k}$,

$$
\left.\widehat{l(F)}:=\widehat{\left(F, L^{*} e\right.}\right)_{\mathcal{H}}=\sum_{i=1}^{q+1} \sum_{j=1}^{M_{i}} \int_{\Omega_{j}^{i}}\left(y_{j}^{i}(t), u_{j}^{i}(t)\right)_{n} d t+c,
$$

where $u_{j}^{i}(t)=\left(U_{j}^{i}\right)^{*} e(t)$ are vector-functions belonging to $\left(L^{2}\left(\Omega_{j}^{i}\right)\right)^{l}, c=(C, e) \in \mathbb{C}$.

By Theorem 2,

$$
\frac{\inf }{\left(F, L^{*} e\right)_{\mathcal{H}}} \sup _{G_{1}, G_{2}} \mathbb{E} \mid\left(F, L^{*} e\right)_{\mathcal{H}}-\left(\left.\widehat{\left.\overline{F, L^{*} e}\right)_{\mathcal{H}}}\right|^{2}=\sup _{G_{1}, G_{2}} \mathbb{E}\left|\left(F, L^{*} e\right)_{\mathcal{H}}-\left(\widehat{\widehat{F, L^{*} e}}\right)_{\mathcal{H}}\right|^{2},\right.
$$

Mathematical Modeling and Computing, Vol. 8, No. 2, pp. 317-329 (2021) 
where $\left(\widehat{\widehat{F, L^{*} e}}\right)_{\mathcal{H}}=\left(\hat{\hat{F}}, L^{*} e\right)_{\mathcal{H}}$ with $\hat{\hat{F}}:=\left(\hat{f}, \hat{g}_{1}, \ldots, \hat{g}_{q}\right)$ and $\hat{f}(\cdot), \hat{g}_{1}, \ldots, \hat{g}_{q}$ being determined by (28). From the latter relationship and from the fact that $\hat{\hat{F}}$ does not depend on $L^{*} e$ (see Remark 1) it follows that vector $\hat{g}=L \hat{F}$ satisfies the assumptions of Lemma 3 . This proves the validity of the following assertion.

Theorem 2. The optimal estimates $\hat{\hat{F}}$ and $\hat{\hat{g}}$ of $F$ and $g=L F$ are determined by $\hat{\hat{F}}=$ $\left(\hat{f}(\cdot), \hat{g}_{1}, \ldots, \hat{g}_{q}\right)$ and $L \hat{\hat{F}}$, respectively, where $\hat{f}(\cdot), \hat{g}_{1}, \ldots, \hat{g}_{q}$ are determined by $(28)$.

Remark 2. All the results of the paper remain valid if we assume that the components $\tilde{\xi}_{j}^{i}(\cdot)$, of random elements $\tilde{\xi}:=\left(\tilde{\xi}_{1}^{1}(\cdot), \ldots, \tilde{\xi}_{M_{1}}^{1}(\cdot), \ldots, \tilde{\xi}_{1}^{q+1}(\cdot), \ldots, \tilde{\xi}_{M_{q+1}}^{q+1}(\cdot)\right)$ entering into the set $G_{2}$ are pairwise uncorrelated and satisfy the condition

$$
\int_{\Omega_{j}^{i}} \operatorname{Tr}\left[D_{j}^{i}(t) \tilde{R}_{j}^{i}(t, t)\right] d t \leqslant 1, \quad i=1, \ldots, q+1, \quad j=1, \ldots, M_{i} .
$$

\section{Conclusion}

The method proposed in the present paper enables one to obtain the optimal estimates of unknown data of periodic boundary value problems for the first-order linear impulsive systems of ordinary differential equations from noisy observations of their solutions.

It has been deduced the periodic boundary value problems for linear impulsive ordinary differential equations of the special kind that generate the optimal estimates.

The results presented above aimed at elaborating mathematically justified estimation techniques for various direct and inverse problems with uncertainties describing periodic evolution processes characterized by the combination of a continuous and abrupt change of their state.

[1] Nakonechny O. G., Podlipenko Yu. K. The minimax approach to the estimation of solutions to first order linear systems of ordinary differential periodic equations with inexact data. Preprint arXiv:1810.07228V1 (2018).

[2] Nakonechnyi O., Podlipenko Y. Guaranteed Estimation of Solutions of First Order Linear Systems of Ordinary Differential Periodic Equations with Inexact Data from Their Indirect Noisy Observations. 2018 IEEE First International Conference on System Analysis \& Intelligent Computing (SAIC). 1-4 (2018).

[3] Samoilenko A. M., Perestyuk N. A. Impulsive differential equations. World Scientific (1995).

[4] Bainov D. D., Simeonov P. S. Impulsive Differential Equations: Periodic Solutions and Applications. World Scientifc (1995).

[5] Rachunkova I., Tomecek J. State-Dependent Impulses. Boundary Value Problems on Compact Interval. Atlantis Press, Amsterdam-Paris-Beijing (2015).

[6] Badriev I. B., Karchevsky M. M. Duality methods in applied problems. Publ. of Kazan State University, Kazan (1987). 


\title{
Оптимальне оцінювання невідомих даних періодичних крайових задач для систем лінійних імпульсних звичайних диференціальних рівнянь першого порядку за непрямими зашумленими спостереженнями ї розв'язків
}

\author{
Наконечний О. Г., Подлипенко Ю. К. \\ Київсъкий начіональний університет імені Тараса Шевченка, \\ вул. Володимирсъка, 64/13, 01601, Київ, Украӥна
}

\begin{abstract}
Розглядаються крайові задачі з періодичними граничними умовами для систем лінійних імпульсних звичайних диференціальних рівнянь першого порядку з невідомими правими частинами та стрибками розв'язків в імпульсних точках, які входять до постановки задач, у припущенні, що вони підпорядковані квадратичним обмеженням. За непрямими зашумленими спостереженнями їх розв'язків на скінченній системі інтервалів отримані оптимальні, у деякому сенсі, оцінки образів цих невідомих даних при їх лінійних неперервних відображеннях. Встановлено, що якщо невідомі кореляційні функції похибок у спостереженнях належать деяким спеціальним множинам, то такі оцінки виражаються через розв'язки деяких періодичних крайових задач для лінійних систем імпульсних звичайних диференціальнх рівнянь.
\end{abstract}

Ключові слова: оптимальні оцінки, гарантовані оцінки, зашумлені спостереження, періодичні крайові задачі, імпульсні звичайні диференціальні рівняння. 